\title{
Mass Spectrometry Based Molecular 3D-Cartography of Plant Metabolites
}

\author{
Dimitrios J. Floros ${ }^{1,2}$, Daniel Petras ${ }^{1,2}$, Clifford A. Kapono ${ }^{1,2}$, Alexey V. Melnik ${ }^{1,2}$, \\ Tie-Jun Ling 1,2,3, Rob Knight ${ }^{4,5,6}$ and Pieter C. Dorrestein ${ }^{1,2,5 *}$
}

'Skaggs School of Pharmacy and Pharmaceutical Sciences, University of California, San Diego, San Diego, CA, USA, ${ }^{2}$ Collaborative Mass Spectrometry Innovation Center, Skaggs School of Pharmacy and Pharmaceutical Sciences, University of California, San Diego, San Diego, CA, USA, ${ }^{3}$ State Key Laboratory of Tea Plant Biology and Utilization, Anhui Agricultural University, Hefei, China, ${ }^{4}$ Department of Computer Science and Engineering, University of California, San Diego, San Diego, CA, USA, ${ }^{5}$ Center for Microbiome Innovation, University of California, San Diego, San Diego, CA, USA, ${ }^{6}$ Department of Pediatrics, University of California, San Diego, San Diego, CA, USA

\section{OPEN ACCESS}

Edited by:

Basil J. Nikolau,

lowa State University, USA

Reviewed by:

Norberto Peporine Lopes,

University of São Paulo, Brazil

Ute Roessner

University of Melbourne, Australia

${ }^{*}$ Correspondence:

Pieter C. Dorrestein

pdorrestein@ucsd.edu

Specialty section:

This article was submitted to Technical Advances in Plant Science, a section of the journal

Frontiers in Plant Science

Received: 24 January 2017

Accepted: 13 March 2017

Published: 29 March 2017

Citation:

Floros DJ, Petras D, Kapono CA,

Melnik AV, Ling T-J, Knight $R$ and

Dorrestein PC (2017) Mass

Spectrometry Based Molecular

3D-Cartography of Plant Metabolites.

Front. Plant Sci. 8:429.

doi: 10.3389/fpls.2017.00429
Plants play an essential part in global carbon fixing through photosynthesis and are the primary food and energy source for humans. Understanding them thoroughly is therefore of highest interest for humanity. Advances in DNA and RNA sequencing and in protein and metabolite analysis allow the systematic description of plant composition at the molecular level. With imaging mass spectrometry, we can now add a spatial level, typically in the micrometer-to-centimeter range, to their compositions, essential for a detailed molecular understanding. Here we present an LC-MS based approach for 3D plant imaging, which is scalable and allows the analysis of entire plants. We applied this approach in a case study to pepper and tomato plants. Together with MS/MS spectra library matching and spectral networking, this non-targeted workflow provides the highest sensitivity and selectivity for the molecular annotations and imaging of plants, laying the foundation for studies of plant metabolism and plant-environment interactions.

Keywords: plant metabolomics, imaging mass spectrometry, 3D-imaging, tomato, pepper

\section{INTRODUCTION}

Phototrophs form the foundational layer of energy and nutrient capture in essentially all terrestrial and marine ecosystems (Field et al., 1998). Human society has long relied on both wild and cultivated plants to provide not only dietary energy, essential micronutrients and medicinally useful natural products, but also structural materials and biofuels (Lewis and Elvin-Lewis, 1995; Bouis, 1996; Durrett et al., 2008). Although many studies have investigated the genetic (Koch, 2016), transcriptomic (Imadi et al., 2015), and proteomic properties (Jorrín-Novo et al., 2015) associated with plant tissues, plant metabolites have long been studied only in the context of bioactive metabolic products. The emerging field of metabolomics is opening the door to a global understanding of plant phenotypes at the molecular level (Sumner et al., 2015). With a quarter of plant genes dedicated to metabolism and over 200 thousand unique natural products identified, the detection and mapping of three dimensional distributions of plant chemistries remains underexplored (Dixon and Strack, 2003). For example, what are the chemical differences between tissue types and growth stages? How do these chemotypes behave in comparison to other species? Are the main factors driving the distribution of chemicals over the plant organ structure, vascularization, 
exposure to sunlight or other environmental gradients as microbial symbionts or pathogens? Understanding these questions at a basic chemical level is an important step to understanding how to engineer plants to better resist erosion and disease, and to improving their efficiency as providers of animal nutrition, feedstocks to industrial processes or the many other roles that plants perform in natural and engineered landscape ecosystems (Yandeau-Nelson et al., 2016).

A wide range of analytical techniques such as NMR and UV or IR spectroscopy, are available for making metabolomic measurements, but suffer from limitations of sensitivity and specificity to individual molecular features (Schripsema, 2010). Unlike spectroscopic techniques, mass spectrometry (MS) allows highly selective and quantitative measurements to be taken of a broad range of chemical families over a large dynamic range. With recent advantages in imaging mass spectrometry (IMS), we can obtain metabolic inventories with spatial information from nm to $\mathrm{km}$ scale (Petras et al., 2017). Matrix assisted laser desorption ionization (MALDI) and laser assisted electrospray ionization (LAESI) use laser shots that raster the surface of a sample to generate molecular 2D images, while desorption based DESI and nano-DESI raster continuously flowing solvent droplets across the sample (Watrous et al., 2012; Etalo et al., 2015; Sturtevant et al., 2016). These techniques vary in their requirements for sample preparation, spatial resolution, and compatibility with atmospheric conditions, but are all limited to $2 \mathrm{D}$ surface imaging. To create $3 \mathrm{D}$ chemical maps, multiple sectioning and reconstruction of $2 \mathrm{D}$ layers is required (as in X-ray tomography or magnet resonance imaging). Apart from these techniques, liquid chromatography based mass spectrometry (LC-MS or LC-MS/MS) methods separate complex components of a tissue extracts based on physiochemical properties before their introduction to the mass spectrometer, mostly via electrospray ionization (ESI). This pre-separation enables greater sensitivity and depth of coverage, making it a powerful tool for metabolomic analyses (De Vos et al., 2007). Another advantage of LC-MS based imaging is that the spatial scale is not limited to instrument based dimensions, typically in the $\mathrm{cm}$ range. This enables the molecular 3D imaging of humans (Bouslimani et al., 2015) and their habitats (Petras et al., 2016) up to planetary ( $\mathrm{km})$ scale. Since the samples are excised, homogenized, and extracted with organic solvents for LC-MS analysis, it is not compatible with direct imaging of plant tissue. However, by generating optical 3D images prior to dissection and recording spatial sampling locations, new visualization tools can map metabolomic features onto the model.

Here we describe an LC-MS based technique for the 3D-mapping of metabolic content of a tomato (Solanum lycopersicum) and pepper plant (Capsicum annuum), two members of the nightshade family. The protocol and analysis pipeline provide a means for spatially mapping the relative intensities of all metabolites detected through an untargeted LCMS/MS metabolomics analysis survey of whole plants. Together with global statistical treatments of the data, these ion maps will help investigators to quickly identify patterns and metabolites of interest in their plant systems.

\section{EXPERIMENTAL CONCEPT}

Our experimental workflow is shown in Figure 1. The initial step of our imaging approach is the creation of optical three dimensional models for the representation of the organism to be sampled. For experiments with little plant to plant variation in size and shape it may be suitable to use a single template to map all samples, in this study we generated models of the specific organisms to be used prior to their dissection. Members of the nightshade family, tomato (S. lycopersicum) and pepper (C. annuum), were therefore purchased from a local nursery. Three dimensional models were generated using a structure from motion software and desktop 3D Scanner to scan the smaller pepper plant. Structure from motion software utilized multiple optical images or photographs from many angles to reconstruct the three dimensional surface of the tomato plant. The tomato plant was placed on a flat surface with minimal background along with several colorful printed reference points to provide consistent points of overlap between photos. The scanner uses infrared lasers as distance finders to accurately capture the surface depth as it is manipulated by a rotating platform, and multiple images are captured and merged by the software. After 3D models had been generated, the aerial plant tissues were dissected, weighed, and quenched in $-20^{\circ} \mathrm{C}$ extraction solvent. Downstream sample preparation involved homogenization and extraction of plant tissues with extraction solvent prior to subsequent evaporation and resuspension for LC-MS/MS analysis. For the LC-MS/MS analysis a 10 min chromatographic separation (UHPLC) method was used, enabling an analysis of 144 samples, e.g., voxel per day. After injecting the samples, molecules were eluted from the reverse phase $\mathrm{C}_{18}$ column with a linear gradient of water and acetonitrile and with $0.1 \%$ formic acid and infused into hybrid quadrupole-time-of-flight (Q-TOF) mass spectrometer. Once acquired, raw vendor data files were recalibrated with an internal standard and converted to mzXML format. Our first step of LC-MS/MS data analysis was to perform a comparison of the presence and intensity of chromatographic ion peaks in extracted ion maps (EIM) (XIC, e.g., retention time $m / z$ pairs). This process, typically called "feature finding," can be time consuming and require substantial computational resources, depending on the data size and algorithms used for feature detection and alignment. MZmine2 (Pluskal et al., 2010), an established tool with easy to use graphical interface was used. After manual inspection of aligned XICs, features were exported as .csv matrix of samples and features with the area under the curve. The feature matrices were used for visualization in principal coordinate analysis (PCoA) with EMPeror (Vazquez-Baeza et al., 2013) and for the generation of 3D EIM using 'ili. Molecular annotation of features was performed in parallel with MS/MS data by spectra library matching and spectral networking using GNPS (Wang et al., 2016). In GNPS, the MScluster algorithm (Frank et al., 2008) is first used to consolidate identical spectra. All consensus spectra are then compared and scored against each other, and organized into a molecular network depending on their spectral similarity. The network was then visualized in Cytoscape 


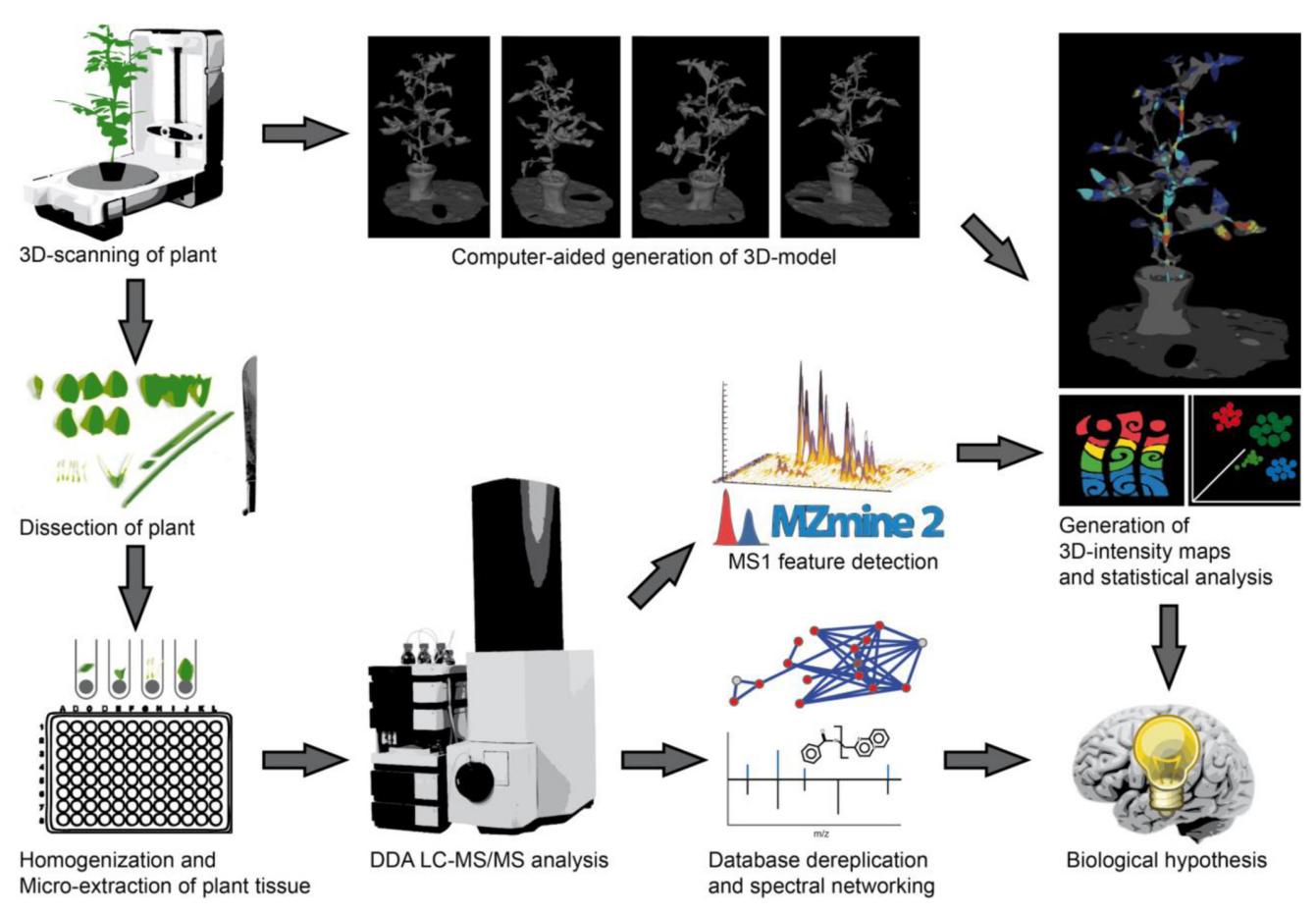

FIGURE 1 | Workflow from plant to chemical 3D ion intensity maps. First, the plant is 3D scanned, either using dedicated hardware (for small and/or complex plants) or structure from motion (for larger and/or less complex plants). The 3D model is then generated and used as a template for analysis. Afterwards, the plant is dissected, and metabolites extracted in 96-well plates. Each sample is analyzed by non-targeted LC-MS/MS with data dependent acquisition (DDA). The MS/MS dataset is then dereplicated through MS/MS spectra database comparison and molecular networking is used to relate MS/MS features to one another and to perform chemical identification through spectral proximity. The MS data is used to identify molecular features, which are further linked to MS/MS based identifications in the molecular network. Finally, the location of each sample is mapped back to the 3D structure of the plant to localize the relative intensity of each molecular feature within this 3D structure. Statistical analyses of MS features such as principal coordinates analysis (PCoA) are performed complementing the explicitly spatial map with a dimensionality-reduced abstract map showing intrinsic similarities and differences in the samples.

(Shannon et al., 2003). Experimental spectra were compared to different reference libraries, including the ReSpect library of phytochemical MS/MS spectra (Sawada et al., 2012). As metabolites with similar fragmentation spectra are organized in network clusters, spectral annotations can be propagated throughout the network to provide putative annotations for previously undescribed molecules. Finally, by assigning colors to the network nodes based on their tissue or organisms of origin, one can quickly generate hypotheses about which metabolite families contain chemically biologically distinctive members, and whether these families are non-randomly distributed among plant locations, tissues or species.

\section{RESULTS AND DISCUSSION}

For an initial global analysis of sample to sample distances, we visualized MS features in the first three dimensions of PCoA space (Figure 2) in EMPeror using the binary Jaccard dissimilarity metric (Vazquez-Baeza et al., 2013). Within this space, tissue type distinctions from the global metabolomic level are readily visible. As expected, leaf, flower, fruit, and stem carry distinct metabolic signatures. Some quantitative distinctions may be driven by differences in extraction efficiency between tissue types, like tough stems and high-water content of tomato flesh. However, the qualitative presence/absence of LC-MS features also varies across tissues. In fact less than $10 \%$ of LC-MS features are observed in all tissue types, suggesting that untargeted analysis of crude extracts are capturing tissue level chemical diversity. In order to determine to what degree the individual metabolites vary between tissue types it is necessary to begin grouping individual metabolites, identifying those that can be dereplicated and observing families of unknown metabolites.

LC-MS features are the first level of mass spectrometric data, but molecular fragmentation (MS/MS) spectra were also collected. These fragmentation patterns contain chemical information about individual metabolites that can be compared using spectra library comparison and spectral networking (Wang et al., 2016). This allows metabolites to be grouped into families of similar compounds as well as being matched against libraries of known compounds (Nguyen et al., 2013). From the resulting molecular network the background nodes, shared with solvent and system blanks, were subtracted, leaving in total 5598 nodes (Figure 3A). Of those, 92 consensus spectra matched thereby to spectra from the libraries within a mass error of $20 \mathrm{ppm}$, yielding a $1.6 \%$ annotation rate. When categorized by tissue type (Figure 3B), MS/MS nodes show similar distributions as MS features (Figure 2), with stem and leaf metabolites making up a 


\section{A Tomato}

\section{B Pepper}

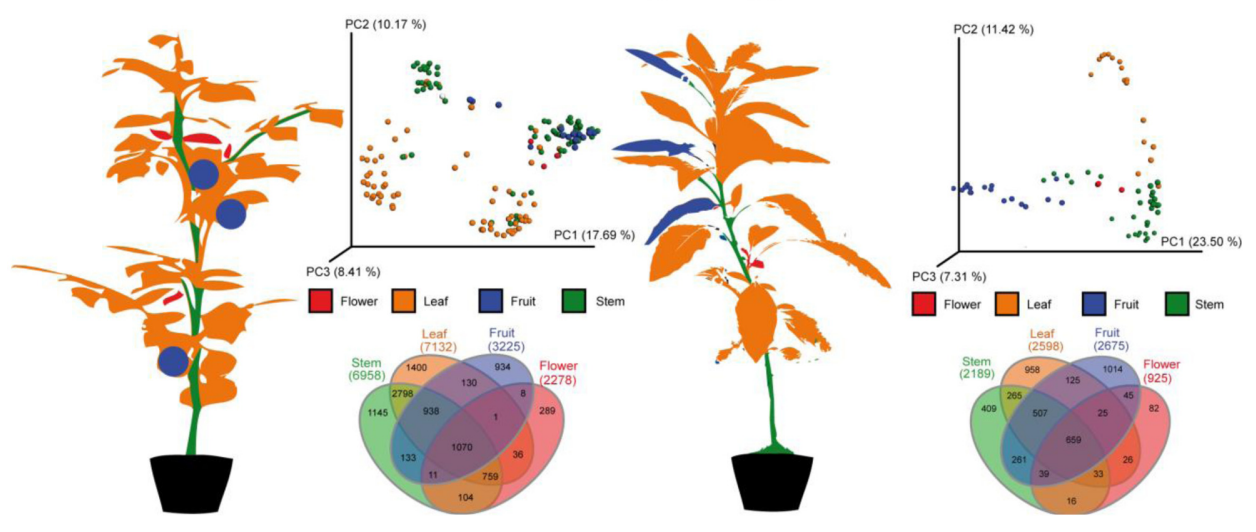

FIGURE 2 | Molecular distribution across the (A) tomato and (B) pepper plants. In each case, a cartoon of the plant showing the individual patches labeled with the same colors used in the principal coordinates plots and Venn diagrams is shown. The principal coordinates plots show the first three principal axes, using the binary Jaccard's distance metric. Samples separate by type and the Venn diagrams demonstrate that only a minority of MS features are shared among all parts of the plant (5.5\% for tomato, $7.9 \%$ for pepper), that stems and leaves share more metabolites than any other pair of samples in both species, and that stems, leaves and fruit each have more unique metabolites than flowers although the rank order of unique metabolites varies between the two plants samples. See Figure $\mathbf{4}$ for the distribution of individual metabolites of interest on the 3D structure of the plant.

large number of total amount of nodes. Fruit and flower tissues contribute many unique signals, while a relatively small number of metabolites are universally detected in all tissues. Matches to library data are present all along this distribution (Figure 3C), providing evidence that the untargeted metabolomics equally sampled a wide range of compounds and that these were represented within the library. Molecular networking performs especially well in identifying uniquely expressed metabolites in a survey (Floros et al., 2016; Nguyen et al., 2016). It is clear that in our molecular network (Figure 3A), with background spectra removed, are multiple molecular families (connected network components) which are either unique to or dominated by samples from specific tissue types.

In Figure $3 \mathrm{C}$ the distribution of the frequency at which metabolites are observed at the MS/MS level ranges from the observations for the internal standard, glycocholic acid, in every sample to pheophytin, chlorophyll without the central $\mathrm{Mg}^{2+}$ ion (Lambers et al., 2008), which was observed in more than 60 samples from stem and leafs, which is not surprising as it forms an essential part of photosynthesis. Chlorogenic acid, on the other hand, an intermediate of the biosynthesis of lignin (Boerjan et al., 2003), an important organic polymer and structural building block of plants, was observed in several redundant nodes, originating from more than 41 samples from stem and leaf. Spectral redundancy can be typically overserved in molecular networks, especially for frequently observed compounds. The reasons therefore can be the differences in abundance of precursors which results in presents or absence of low intensity MS/MS fragments and thus classification in different consensus spectra. The flavonoid, kaempferol-3-O-glucoside, a typical phytochemical which has been studied in the context of antioxidant and anti-inflammatory activity (Parveen et al., 2007) was present in total 34 samples, but mostly in leaves. On the low frequent side, nodes matching to spectra from capsaicin were only observed in eight samples originating from fruits from the pepper plant.

Once data at the global levels has indicated that tissue level distinctions and tissue specific metabolite families are present, the visualization of individual metabolites was achieved with the generation of EIMs (Figure 4). Visualization in 'ili allows investigators to cycle through the distributions of each individual metabolite. This takes advantage of the brain's enormous capacity to absorb visual information and detect patterns; EIMs allow rapid hypothesis generation and potential biomarker selection. Comparing single metabolite patterns to known physiological and pathological patterns can provide insight into the biological function of certain metabolites in many systems. Figure 4 shows exemplary EIMs of the above-mentioned metabolite features. Once present in 'ili, the creation of an EIM can be performed instantly with every molecular feature present in the .csv file. In Figure 4A, the distribution of tri-coumaroyl spermidine can be seen. The strongest abundance can be overserved at both, the flowers of the pepper and the tomato plant. These findings are in line with the literature, where coumaroylated spermidines have been reported to be common in floral organs (Werner et al., 1995), in particular in tomatoes (Larbat et al., 2014). Phenolic amines, such as tri-coumaroylated spermidine, have thereby been studied mainly in the context of plant defense and biotic aggression (Elejalde-Palmett et al., 2015) which might be an important molecular factor for future crop breeding. In Figure 4B the spatial distribution of tryptophan, a canonical amino acid, is shown. As tryptophan is common in basically all living organism it's detection is not too surprising. However, tryptophan is a biosynthetic precursor of indole acetic acid an auxin important for fruit development (Ljung et al., 2002). Hence, it's interesting to observe that the relative concentration of tryptophan shows the highest abundance in the tomato and pepper fruits, which can be important to understand differences 
A

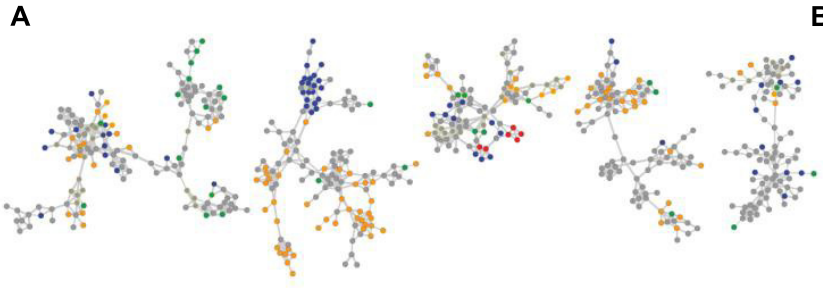

B

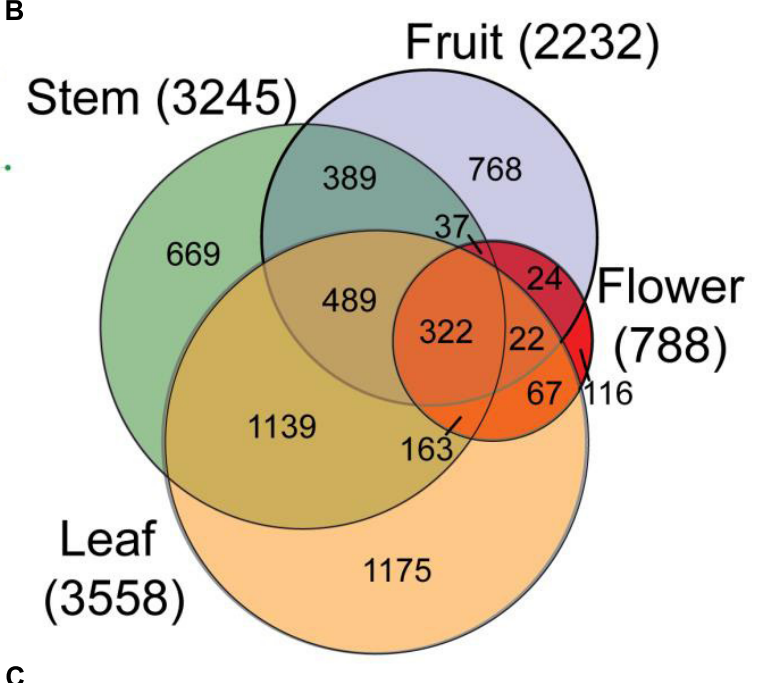

4

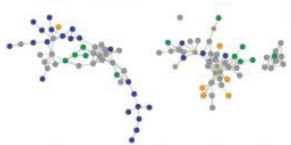

$\cdots$

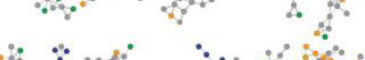

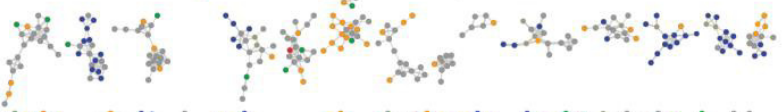

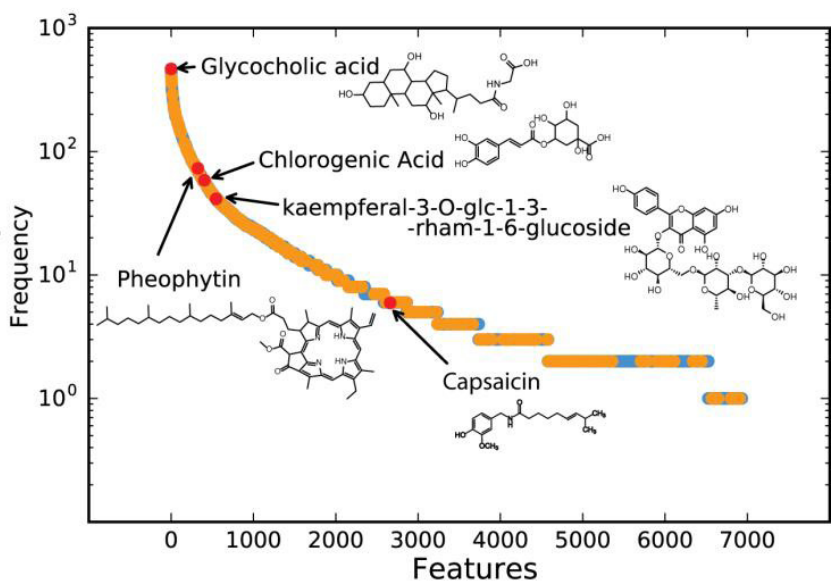

-

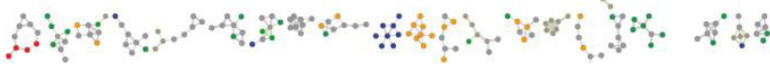
की

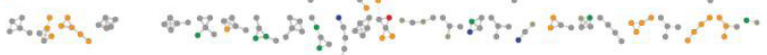

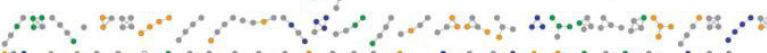

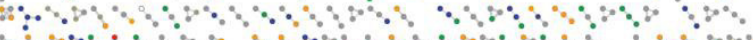

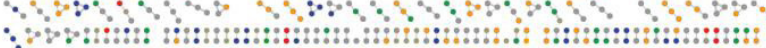
:
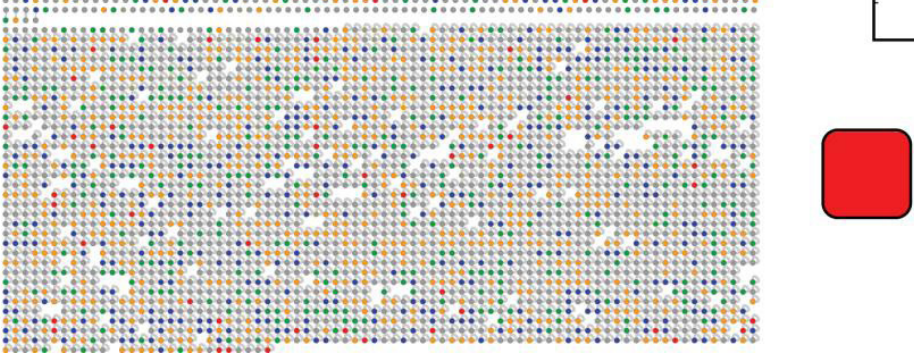
Flower
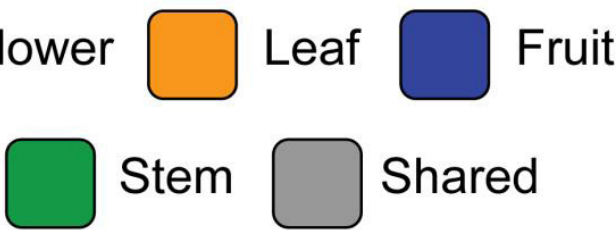

Stem

Shared

FIGURE 3 | Molecular MS/MS network and metabolite level overlap. (A) Product ion spectra of all tissues samples, were analyzed based on their spectral proximity with GNPS. Every node represents a single or set of identical spectra, linked to similar MS/MS spectra. The different tissue origins of the spectra are indicated by different node colors. Nodes originating from solvent and/or system blanks were subtracted. In (B) Venn diagrams of the occurrence of unique and shared MS/MS spectra between different tissue types are shown. In (C) the frequency plot of clusters of MS/MS spectra (represented as nodes in the network) vs samples in which this MS/MS spectrum is observed.

in fruit maturing processes, particularly of agricultural important species. Figure $4 \mathrm{C}$ shows the spatial distribution of capsaicin which was found only in the fruit of the pepper plant. In Figure 4D the spatial distribution of an unknown metabolite with the mass $446.2480 \mathrm{~m} / \mathrm{z}$ is shown which is only observed in the tomato. Although it is unknown now, it might be identified and automatically annotated through the "living data" in GNPS or could be subjected to further targeted structural and functional investigations.
Summarizing, LC-MS based 3D imaging of plants introduces a new tool to spatially visualize small molecules in plants and thus to understand phenotypic and genetic differences on a metabolite level. Paired with spatial high resolution imaging methods such as MALDI and other modalities, we anticipate this technique will provide the base for most comprehensive molecular models of complete plants and their interactions with their environment and microorganisms. 

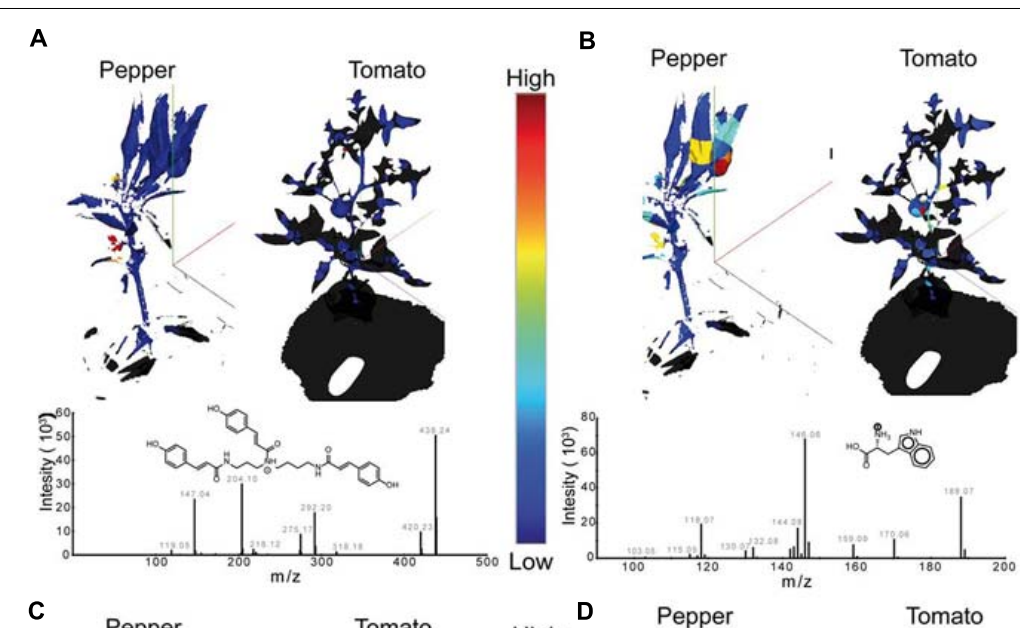

C Pepper

D Pepper

Tomato
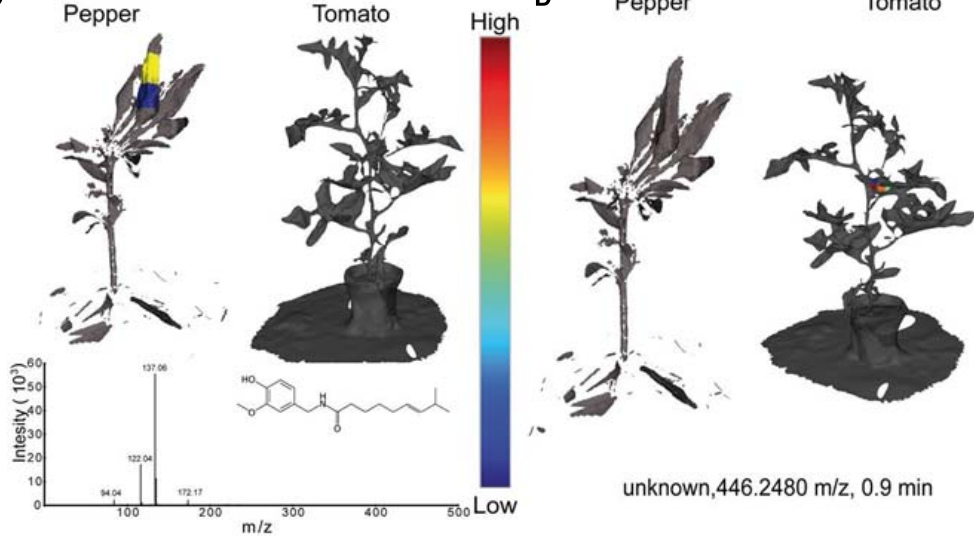

FIGURE 4 | Extracted 3D ion maps of a pepper and tomato plant. The spatial distribution of (A) tri-coumaroyl- spermidine, (B) tryptophan, (C) Capsaicin, and (D) an unknown compound are shown. Relative concentrations of each $\mathrm{m} / \mathrm{z}$ - retention-time feature are displayed with a linear color gradient (blue - red) in the extracted ion maps of both plants. All MS features detected in our study can be found in the supporting information and can be visualized by drag and drop of the .stl and .csv files in 'ili (ili-toolbox.github.io).

\section{EXPERIMENTAL PROCEDURE}

\section{Plants}

Two individual plants, tomato ( $S$. lycopersicum) and pepper (C. annuum), were purchased from a local nursery (Green Gardens Nursery, San Diego, CA, USA) in October 2015.

\section{Model Generation}

3D models were generated using the structure-from-motion software 123Dcatch (Autodesk, San Rafael, CA, USA) for the larger tomato plant, and the 3D Scanner Ultra HD (NextEngine, Santa Monica, CA, USA) for portions of the pepper plant. Both platforms provide output file in stl formats, which are then used with the 'ili toolbox.

\section{Sectioning, Processing, and Extraction}

All aerial plant tissues parts were dissected manually into sections with a size convenient for extraction in a $1.5 \mathrm{~mL}$ reaction tube. Each tissue section was weighed, with masses ranging from 0.01 to 0.03 grams. Tissues were placed in round-bottom solvent resistant tubes along with a $5 \mathrm{~mm}$ stainless steel bead and 500 microliters of extraction solvent (2:2:1 ethyl acetate:methanol:water) and were disrupted by shaking at $30 \mathrm{~Hz}$ for $15 \mathrm{~min}$ in a TissueLyser (Qiagen, Hilden, Germany). Plant tissues were allowed to extract for at least $8 \mathrm{~h}$ at $-20^{\circ} \mathrm{C}$. Tubes were then centrifuged briefly to pellet solid material and the supernatants were transferred to $1 \mathrm{~mL}$ deep 96-well plates and stored at $-20^{\circ} \mathrm{C}$ until being evaporated to dryness in a Speedvac vacuum centrifuge (Thermo, Bremen, Germany). Samples were then resuspended in injection solvent (9:1 methanol:water containing $10 \mathrm{mM}$ glycocholic acid as internal standard) by sonication of the entire plate for $5 \mathrm{~min}$. Again, insoluble particulates were pelleted by centrifugation at $20,000 \mathrm{~g}$ for 10 min. Finally, 100 microliters of extracted solvent was transferred to polypropylene $0.3 \mathrm{~mL}$ 96-well plates (NUNC, Roskilde, Denmark). Plates were stored at $-80^{\circ} \mathrm{C}$ until LC-MS analysis.

\section{LC-MS Analysis}

All sample extracts were subjected to chromatographic separation with an Agilent 1290 Infinity UHPLC system (Agilent, 
Waldbronn, Germany). Separations were achieved using a flow rate of $0.5 \mathrm{~mL} / \mathrm{min}$ with an 1.7 micron $\mathrm{C} 18(50 \times 2.1 \mathrm{~mm})$ Kinetex UHPLC column (Phenomenex, Torrance, CA, USA) held at $30^{\circ} \mathrm{C}$. The gradient was $0-0.5 \mathrm{~min} 5 \% \mathrm{~B}, 0.5-8 \mathrm{~min}$ $5-100 \%$ B, 8-11 min $100 \%$ B, 11-11.5 min 100-5\% B, 11.512 min $5 \% \mathrm{~B}$, where solvent $\mathrm{A}$ is water with $0.1 \%$ formic acid $(\mathrm{v} / \mathrm{v})$ and solvent B is acetonitrile with $0.1 \%$ formic acid $(\mathrm{v} / \mathrm{v})$. MS/MS experiments were performed on a Maxis QTOF mass spectrometer (Bruker, Bremen, Germany) equipped with an ESI source in positive mode with mass range $100-2000 \mathrm{~m} / \mathrm{z}$. Before analysis, the instrument was externally calibrated to 1.0 ppm mass accuracy with ESI-L Low Concentration Tuning Mix (Agilent, Waldbronn, Germany). During the analysis $\mathrm{m} / \mathrm{z}$ 622.029509, was used as an internal calibrant. Instrument parameters were as follows: nebulizer gas $\left(\mathrm{N}_{2}\right)$ pressure, two bar; Capillary voltage, $4500 \mathrm{~V}$; ion source temperature $200^{\circ} \mathrm{C}$; dry gas $9 \mathrm{~L} / \mathrm{min}$; spectra rate, $2 \mathrm{~Hz}$. MS/MS fragmentation of the five most abundant ions per spectrum was performed with adaptive collision energy and acquisition time based on precursor ion properties (see Supplementary Materials). With total cycle time of $3 \mathrm{~s}$, ions were excluded from reselection after three spectra and released after $20 \mathrm{~s}$. An exclusion list was used to prevent sampling of the lock mass. All LC-MS analyses were controlled by Hystar and Otof Control software packages (Bruker, Bremen, Germany). Raw data was then converted to .mzXML format.

\section{Data Processing and Multivariable Statistical Analysis}

For the global statistical analysis, the MS/MS scans were removed from the original mzXML files using MSConvert (Deutsch et al., 2015) to decrease the total file and speed up down-stream processing. Feature extraction was performed with MZmine2 (Pluskal et al., 2010) with a signal threshold of $2.0 \mathrm{e}^{6}$ and $0.3 \mathrm{~s}$ minimum peak width. The mass tolerance was set to $10 \mathrm{ppm}$ and the maximum allowed retention time deviation was set to 10 s. For chromatographic deconvolution the baseline cutoff algorithm with 1.0e4 signal threshold was used. Maximum peak width was set to $2 \mathrm{~min}$. After isotope peak removal, the peak lists of all samples were aligned with the above-mentioned retention time and mass tolerances. After the creation of a feature matrix containing the feature retention times, exact mass and peak areas of the corresponding extracted ion chromatograms, metadata of the samples (plant type, tissue type, and spatial coordinates) was added. For the PCoA the signal intensity of the features was normalized to the total ion current of all samples. The PCoA plots were generated with the binary Jaccard dissimilarity metric using the in-house tool ClusterApp and visualized in EMPeror (Vazquez-Baeza et al., 2013). Venn diagrams of shared MS1 features were generated using InteractiVenn (Heberle et al., 2015).

\section{Molecular Networking}

MS/MS data was analyzed with GNPS (Wang et al., 2016). Therefore, the data was filtered by removing all MS/MS peaks within a $17 \mathrm{Da}$ window of the precursor $\mathrm{m} / \mathrm{z}$ and MS/MS spectra were filtered by choosing only the top 6 peaks in $50 \mathrm{Da}$ windows. The data was then clustered with MS-Cluster (Frank et al.,
2008), with a precursor mass tolerance of $0.02 \mathrm{Da}$ and a MS/MS fragment ion tolerance of $0.02 \mathrm{Da}$. Consensus spectra with less than 2 spectra were discarded. A spectral network was then created with a minimum spectral similarity of cosine 0.7 and more than 4 matched peaks. Only top 10 edged connecting one note were kept in the network. Consensus spectra were searched against the GNPS spectral library as well as Massbank, ReSpect, HMDB, and NIST14 (Forsythe and Wishart, 2009; Horai et al., 2010; Sawada et al., 2012; Stein, 2014; Wang et al., 2016) with a precursor mass tolerance of $0.02 \mathrm{Da}$ and a MS/MS fragment ion tolerance of $0.02 \mathrm{Da}$. Nodes originating from solvent and/or system blanks were subtracted. Venn/Euler diagrams of MS/MS features from GNPS were generated using the Venn and Euler Diagrams app (Heuer et al., 2016) in the Cytoscape 3 environment (Shannon et al., 2003).

\section{D Mapping and Visual Analysis}

We used the GeoMagic 3D visualization and editing software (3D Systems, Rock Hill, SC, USA) to clean up the automatically generated 3D mesh objects from the .stl-format files. This software was also used to identify coordinates corresponding to each tissue sample. Each pair of filenames and coordinates was associated with their corresponding MS features and MS/MS IDs in a .csv-format table. These tables were uploaded, along with the .stl 3D model, to the visualization tool 'ili (Alexandrov, 2016).

\section{DATA ACCESSIBILITY}

All LC-MS/MS data can be found on the Mass spectrometry Interactive Virtual Environment (MassIVE) at https://massive. ucsd.edu/ with the identifiers MSV000079448, MSV000079447, and MSV000079446. 3D .stl files and corresponding ion intensities in .csv format are available in the supporting information of this article.

\section{AUTHOR CONTRIBUTIONS}

DF, DP, and PD designed the study. DF, AM, CK, and T-JL collected the samples. DF and AM acquired the MS data. $\mathrm{DF}, \mathrm{DP}, \mathrm{CK}$, and $\mathrm{AM}$ performed the $3 \mathrm{D}$ mapping. DF and DP processed and analyzed the data. DF, DP, RK, and PD wrote the manuscript. All authors discussed and approved the manuscript.

\section{ACKNOWLEDGMENT}

This work was supported by NSF Inspire Track II IOS-1343020 and NIH S10RR029121 and P41GM103484.

\section{SUPPLEMENTARY MATERIAL}

The Supplementary Material for this article can be found online at: http://journal.frontiersin.org/article/10.3389/fpls.2017.00429/ full\#supplementary-material 


\section{REFERENCES}

Alexandrov, T. (2016). Ili-Toolbox/ili. Available at: https://github.com/ili-toolbox/ ili [accessed April 27, 2016].

Boerjan, W., Ralph, J., and Baucher, M. (2003). Lignin biosynthesis. Annu. Rev. Plant Biol. 54, 519-546. doi: 10.1146/annurev.arplant.54.031902.134938

Bouis, H. (1996). Enrichment of food staples through plant breeding: a new strategy for fighting micronutrient malnutrition. Nutr. Rev. 54, 131-137. doi: 10.1111/j. 1753-4887.1996.tb03915.x

Bouslimani, A., Porto, C., Rath, C. M., Wang, M., Guo, Y., Gonzalez, A., et al. (2015). Molecular cartography of the human skin surface in 3D. Proc. Natl. Acad. Sci. U.S.A. 112, E2120-E2129. doi: 10.1073/pnas.1424409112

De Vos, R. C., Moco, S., Lommen, A., Keurentjes, J. J., Bino, R. J., and Hall, R. D. (2007). Untargeted large-scale plant metabolomics using liquid chromatography coupled to mass spectrometry. Nat. Protoc. 2, 778-791. doi: $10.1038 /$ nprot.2007.95

Deutsch, E. W., Mendoza, L., Shteynberg, D., Slagel, J., Sun, Z., and Moritz, R. L. (2015). Trans-Proteomic Pipeline, a standardized data processing pipeline for large-scale reproducible proteomics informatics. Proteomics Clin. Appl. 9, 745-754. doi: 10.1002/prca.201400164

Dixon, R. A., and Strack, D. (2003). Phytochemistry meets genome analysis, and beyond. Phytochemistry 62, 815-816. doi: 10.1016/S0031-9422(02) 00712-4

Durrett, T. P., Benning, C., and Ohlrogge, J. (2008). Plant triacylglycerols as feedstocks for the production of biofuels. Plant J. 54, 593-607. doi: 10.1111/j. 1365-313X.2008.03442.x

Elejalde-Palmett, C., de Bernonville, T. D., Glevarec, G., Pichon, O., Papon, N., Courdavault, V., et al. (2015). Characterization of a spermidine hydroxycinnamoyltransferase in Malus domestica highlights the evolutionary conservation of trihydroxycinnamoyl spermidines in pollen coat of core Eudicotyledons. J. Exp. Bot. 66, 7271-7285. doi: 10.1093/jxb/erv423

Etalo, D. W., De Vos, R. C., Joosten, M. H., and Hall, R. D. (2015). Spatially resolved plant metabolomics: some potentials and limitations of laser-ablation electrospray ionization mass spectrometry metabolite imaging. Plant Physiol. 169, 1424-1435. doi: 10.1104/pp.15.01176

Field, C. B., Behrenfeld, M. J., Randerson, J. T., and Falkowski, P. (1998). Primary production of the biosphere: integrating terrestrial and oceanic components. Science 281, 237-240. doi: 10.1126/science.281.5374.237

Floros, D. J., Jensen, P. R., Dorrestein, P. C., and Koyama, N. (2016). A metabolomics guided exploration of marine natural product chemical space. Metabolomics 12, 145. doi: 10.1007/s11306-016-1087-5

Forsythe, I. J., and Wishart, D. S. (2009). Exploring human metabolites using the human metabolome database. Curr. Protoc. Bioinformatics Chapter 14, Unit14.8. doi: 10.1002/0471250953.bi1408s25

Frank, A. M., Bandeira, N., Shen, Z., Tanner, S., Briggs, S. P., Smith, R. D., et al. (2008). Clustering millions of tandem mass spectra. J. Proteome Res. 7, 113-122. doi: $10.1021 /$ pr070361e

Heberle, H., Meirelles, G. V., da Silva, F. R., Telles, G. P., and Minghim, R. (2015). InteractiVenn: a web-based tool for the analysis of sets through Venn diagrams. BMC Bioinformatics 16:1. doi: 10.1186/s12859-015-0611-3

Heuer, M., Smoot, M., and Venn and Euler Diagrams. (2016). Available at: http://apps.cytoscape.org/apps/vennandeulerdiagrams [accessed Apr 27, 2016]

Horai, H., Arita, M., Kanaya, S., Nihei, Y., Ikeda, T., Suwa, K., et al. (2010). MassBank: a public repository for sharing mass spectral data for life sciences. J. Mass Spectrom. 45, 703-714. doi: 10.1002/jms.1777

Imadi, S. R., Kazi, A. G., Ahanger, M. A., Gucel, S., and Ahmad, P. (2015). Plant transcriptomics and responses to environmental stress: an overview. J. Genet. 94, 525-537. doi: 10.1007/s12041-015-0545-6

Jorrín-Novo, J. V., Pascual, J., Sánchez-Lucas, R., Romero-Rodríguez, M. C. Rodríguez-Ortega, M. J., Lenz, C., et al. (2015). Fourteen years of plant proteomics reflected in Proteomics: moving from model species and 2DE-based approaches to orphan species and gel-free platforms. Proteomics 15, 1089-1112. doi: $10.1002 /$ pmic. 201400349

Koch, L. (2016). Plant genomics: 1001 genomes and epigenomes. Nat. Rev. Genet. 17, 503-503. doi: 10.1038/nrg.2016.99

Lambers, H., Chapin, I. I. I. F. S., and Pons, T. L. (eds) (2008). "Photosynthesis," in Plant Physiological Ecology, (New York, NY: Springer), 11-99. doi: 10.1007/ 978-0-387-78341-3_2
Larbat, R., Paris, C., Le Bot, J., and Adamowicz, S. (2014). Phenolic characterization and variability in leaves, stems and roots of Micro-Tom and patio tomatoes, in response to nitrogen limitation. Plant Sci. 224, 62-73. doi: 10.1016/j.plantsci. 2014.04.010

Lewis, W. H., and Elvin-Lewis, M. P. (1995). Medicinal plants as sources of new therapeutics. Ann. Mo. Bot. Gard. 82, 16-24. doi: 10.2307/2399976

Ljung, K., Hull, A. K., Kowalczyk, M., Marchant, A., Celenza, J., Cohen, J. D., et al. (2002). "Biosynthesis, conjugation, catabolism and homeostasis of indole3-acetic acid in Arabidopsis thaliana," in Auxin Molecular Biology, eds C. P. Rechenmann and G. Hagen (New York, NY: Springer), 249-272.

Nguyen, D. D., Melnik, A. V., Koyama, N., Lu, X., Schorn, M., Fang, J., et al. (2016). Indexing the Pseudomonas specialized metabolome enabled the discovery of poaeamide B and the bananamides. Nat. Microbiol. 2:16197. doi: 10.1038/ nmicrobiol.2016.197

Nguyen, D. D., Wu, C. H., Moree, W. J., Lamsa, A., Medema, M. H., Zhao, X., et al. (2013). MS/MS networking guided analysis of molecule and gene cluster families. Proc. Natl. Acad. Sci. U.S.A. 110, E2611-E2620. doi: 10.1073/pnas. 1303471110

Parveen, Z., Deng, Y., Saeed, M. K., Dai, R., Ahamad, W., and Yu, Y. H. (2007). Antiinflammatory and analgesic activities of Thesium chinense Turcz extracts and its major flavonoids, kaempferol and kaempferol-3-O-glucoside. Yakugaku Zasshi 127, 1275-1279. doi: 10.1248/yakushi.127.1275

Petras, D., Jarmusch, A. K., and Dorrestein, P. C. (2017). From single cells to our planet-Recent advances in using mass spectrometry for spatially resolved metabolomics. Curr. Opin. Chem. Biol. 36, 24-31. doi: 10.1016/j.cbpa.2016. 12.018

Petras, D., Nothias, L. F., Quinn, R. A., Alexandrov, T., Bandeira, N., Bouslimani, A., et al. (2016). Mass spectrometry-based visualization of molecules associated with human habitats. Anal. Chem. 88, 10775-10784. doi: 10.1021/acs.analchem.6b03456

Pluskal, T., Castillo, S., Villar-Briones, A., and Oresic, M. (2010). MZmine 2: modular framework for processing, visualizing, and analyzing mass spectrometry-based molecular profile data. BMC Bioinformatics 11:395 doi: 10.1186/1471-2105-11-395

Sawada, Y., Nakabayashi, R., Yamada, Y., Suzuki, M., Sato, M., Sakata, A., et al. (2012). RIKEN tandem mass spectral database (ReSpect) for phytochemicals: a plant-specific MS/MS-based data resource and database. Phytochemistry 82 , 38-45. doi: 10.1016/j.phytochem.2012.07.007

Schripsema, J. (2010). Application of NMR in plant metabolomics: techniques, problems and prospects. Phytochem. Anal. 21, 14-21. doi: 10.1002/pca.1185

Shannon, P., Markiel, A., Ozier, O., Baliga, N. S., Wang, J. T., Ramage, D., et al. (2003). Cytoscape: a software environment for integrated models of biomolecular interaction networks. Genome Res. 13, 2498-2504. doi: 10.1101/ gr.1239303

Stein, S. (2014). The NIST 14 Mass Spectral Library. Gaithersburg, MD: National Institute of Standards and Technology.

Sturtevant, D., Lee, Y.-J., and Chapman, K. D. (2016). Matrix assisted laser desorption/ionization-mass spectrometry imaging (MALDI-MSI) for direct visualization of plant metabolites in situ. Curr. Opin. Biotechnol. 37, 53-60. doi: 10.1016/j.copbio.2015.10.004

Sumner, L. W., Lei, Z., Nikolau, B. J., and Saito, K. (2015). Modern plant metabolomics: advanced natural product gene discoveries, improved technologies, and future prospects. Nat. Prod. Rep. 32, 212-229. doi: 10.1039/ c4np00072b

Vazquez-Baeza, Y., Pirrung, M., Gonzalez, A., and Knight, R. (2013). EMPeror: a tool for visualizing high-throughput microbial community data. Gigascience 2:16. doi: 10.1186/2047-217X-2-16

Wang, M., Carver, J. J., Phelan, V. V., Sanchez, L. M., Garg, N., Peng, Y., et al. (2016). Sharing and community curation of mass spectrometry data with global natural products social molecular networking. Nat. Biotechnol. 34, 828-837. doi: $10.1038 /$ nbt.3597

Watrous, J., Roach, P., Alexandrov, T., Heath, B. S., Yang, J. Y., Kersten, R. D., et al. (2012). Mass spectral molecular networking of living microbial colonies. Proc. Natl. Acad. Sci. U.S.A. 109, E1743-E1752. doi: 10.1073/pnas.1203689109

Werner, C., Hu, W., Lorenzi-Riatsch, A., and Hesse, M. (1995). Dicoumaroylspermidines and tri-coumaroylspermidines in anthers of different species of the genus Aphelandra. Phytochemistry 40, 461-465. doi: 10.1016/0031-9422(95)00288-I 
Yandeau-Nelson, M., Lauter, N., and Zabotina, O. (2016). Advances in metabolomic applications in plant genetics and breeding). CAB Rev. 10, $1-15$.

Conflict of Interest Statement: The authors declare that the research was conducted in the absence of any commercial or financial relationships that could be construed as a potential conflict of interest.
Copyright (C) 2017 Floros, Petras, Kapono, Melnik, Ling, Knight and Dorrestein. This is an open-access article distributed under the terms of the Creative Commons Attribution License (CC BY). The use, distribution or reproduction in other forums is permitted, provided the original author(s) or licensor are credited and that the original publication in this journal is cited, in accordance with accepted academic practice. No use, distribution or reproduction is permitted which does not comply with these terms. 